\title{
TERAPÊUTICA INTERDISCIPLINAR PARA FRATURA COMINUTIVA DE CÔNDILO POR PROJÉTIL DE ARMA DE FOGO - ENFOQUE MIOFUNCIONAL
}

\section{Interdisciplinary approach for comminuted condyle fracture of by firearms - myofunctional focus}

\author{
Esther Mandelbaum Gonçalves Bianchini (1), Rogério Bonfante Moraes (2), \\ Daniella Nazario ${ }^{(3)}$, João Gualberto de Cerqueira Luz ${ }^{(4)}$
}

\begin{abstract}
RESUMO
Tema: ferimentos causados por projéteis de arma de fogo apresentam alta incidência na região da cabeça e face. A articulação temporomandibular pode estar envolvida, além de estruturas anatômicas importantes como o nervo facial, necessitando de equipe multidisciplinar para efetuar tratamento adequado. Procedimentos: apresentação de caso clínico de fratura condilar cominutiva causada por projétil de arma de fogo tratado de forma não-cirúrgica associado à terapia miofuncional orofacial. Paciente encaminhado para avaliação e procedimentos fonoaudiológicos após conduta da equipe de cirurgia bucomaxilofacial, sem remoção do projétil, alojado superficialmente, próximo da origem do músculo esternocleidomastóideo à direita, com fratura condilar cominutiva e lesão do nervo facial. Foram aspectos observados em avaliação: mordida aberta anterior, importante redução da amplitude dos movimentos mandibulares com desvios para o lado acometido, ausência de lateralidade contralateral, dor muscular, paralisia e parestesia em terço médio e superior da hemiface direita. Realizadas sessões de terapia miofuncional seguindo protocolo específico para traumas de face constando de: drenagem de edema; manipulações na musculatura levantadora da mandíbula ipsilateral; ampliação e correção dos movimentos mandibulares; procedimentos específicos referentes à paralisia facial e reorganização funcional direcionada. Resultados: após oito semanas de terapia os resultados obtidos mostram restabelecimento de amplitude e da simetria dos movimentos mandibulares, reorganização da mastigação, adequação da deglutição e fala, remissão da sintomatologia dolorosa e remissão da paralisia do terço médio. Conclusão: o tratamento conservador da fratura por meio da terapia miofuncional orofacial resultou na reabilitação funcional da mandíbula e face dirigindo os movimentos e estimulando a adequação das funções estomatognáticas.
\end{abstract}

DESCRITORES: Articulação Temporomandibular; Fraturas Cominutivas; Ferimentos por Arma de Fogo; Reabilitação; Terapia Miofuncional

(1) Fonoaudióloga; Professor Adjunto Mestrado Profissionalizante em Fonoaudiologia da Universidade Veiga de Almeida - UVA, Rio de Janeiro; Coordenadora do Departamento de Distúrbios da ATM, Cirurgia Ortognática e Traumas de face do Instituto CEFAC, São Paulo; Doutora em Ciências - Fisiopatologia Experimental pela Faculdade de Medicina da Universidade de São Paulo - FMUSP.

(2) Cirurgião-Dentista; Mestre em Cirurgia e Traumatologia Buco-maxilo-facial - Faculdade de Odontologia da Universidade de São Paulo - FOUSP.

(3) Fonoaudióloga; Especialista em Motricidade Orofacial.

(4) Cirurgião-Dentista; Professor Associado do Departamento de Cirurgia Prótese e Traumatologia Maxilo-Faciais da

\section{INTRODUÇÃO}

Os ferimentos causados por projéteis de arma de fogo apresentam alta incidência na região da cabeça e/ou face ${ }^{1-4} \mathrm{e}$, muitas vezes a articulação

Faculdade de Odontologia da Universidade de São Paulo FOUSP; Coordenador do curso de Especialização em Cirurgia e Traumatologia Buco-maxilo-facial da Associação Paulista de Cirurgiões Dentista de São Paulo; Livre-Docência pela Universidade de São Paulo.

Conflito de interesses: inexistente 
temporomandibular (ATM) pode estar envolvida, além de estruturas anatômicas importantes como o nervo facial ${ }^{5}$, podendo originar paralisia facial traumática ${ }^{6}$. Esse tipo de lesão apresenta padrão extremamente variável, podendo acometer estruturas vitais e gerar hemorragias de difícil controle, necessitando de equipe multidisciplinar para efetuar o tratamento inicial adequado. Estes ferimentos geralmente resultam em fraturas cominutivas com pequenos e múltiplos fragmentos ósseos ${ }^{1,7}$. Os pacientes acometidos por estas fraturas podem apresentar complicações como desvio mandibular em abertura máxima e em protrusão, limitação da abertura da boca e de lateralidade, mordida aberta anterior, e mais raramente anquilose temporomandibular ${ }^{7}$. As complicações funcionais mais comuns associadas à presença de fratura cominutiva do côndilo incluem limitação parcial dos movimentos mandibulares com desvios destes para o lado da fratura; mastigação unilateral do lado afetado com redução da amplitude do movimento e utilização excessiva de língua amassando o alimento contra o palato; interferência na deglutição devido à insuficiência do preparo do bolo alimentar; articulação da fala com desvio no percurso mandibular também para o lado acometido, uma vez que apenas o movimento condilar do lado oposto está preservado. $O$ repouso mandibular pode estar assimétrico com apoio de língua preferencialmente do lado da fratura ${ }^{8-10}$.

Há grande controvérsia quanto à modalidade de tratamento que deve ser instituída para as fraturas cominutivas de côndilos mandibulares causadas por projéteis de arma de fogo. Algumas modalidades de tratamento são propostas, geralmente seguindo duas tendências distintas: o tratamento cirúrgico, por meio de fixação dos seguimentos fraturados ou substituição por enxertos; e o tratamento conservador não cirúrgico, constando de curto período de imobilização com arco de Erich ou amarrias interdentais, seguido de reabilitação miofuncional orofacial $7^{7-9,11-13}$. Estudos têm demonstrado que 0 tratamento não-cirúrgico para as fraturas condilares por projétil de arma de fogo obtém maior êxito, com menor morbidade para 0 paciente ${ }^{14}$, porém pode resultar em limitação importante da abertura da boca quando tratados sem mobilização imediata ou reabilitação miofuncional ${ }^{7,11,15}$.

A terapêutica fonoaudiológica miofuncional orofacial após esse tipo de trauma de face pode ser indicada, tanto associada aos procedimentos cirúrgicos, quanto aos conservadores. Seus objetivos referem-se principalmente a: aumento da amplitude da abertura da boca, adequação dos movimentos evitando adaptações nocivas e promovendo simetria de mobilidade da face e estabilidade funcional $8,9,15$. Em especial nos casos de fraturas mandibulares, a intervenção miofuncional orofacial visa restabelecer a mobilidade mandibular, evitando possíveis assimetrias, limitações funcionais e sequelas importantes tais como a anquilose temporomandibular. Principalmente nas fraturas altas de côndilo, a reabilitação miofuncional busca a remodelação óssea por força e ação da musculatura envolvida e vem sendo preconizada como tratamento conservador dessas fraturas ${ }^{8,9,11,15}$.

Este trabalho teve por objetivo apresentar os procedimentos e resultados obtidos no tratamento não-cirúrgico associado à terapia miofuncional orofacial, de um caso clínico de fratura condilar cominutiva, causada por projétil de arma de fogo.

\section{APRESENTAÇÃO DO CASO}

Trata-se de relato de caso clínico, com dados da avaliação e procedimentos hospitalares, da avaliação fonoaudiológica e descrição das técnicas de terapia fonoaudiológica miofuncional utilizadas durante atendimento realizado, assim como dos resultados obtidos.

O levantamento dos dados desse trabalho foi iniciado após procedimentos éticos pertinentes: Avaliação e aprovação pelo Comitê de Ética em Pesquisa da Instituição de origem, sob o protocolo número 208/06 e assinatura do Termo de Consentimento Livre e Esclarecido.

Paciente de 20 anos, masculino, leucoderma, vítima de ferimento por projétil de arma de fogo na região da ATM direita, foi atendido no pronto-socorro do Hospital Municipal Dr. Arthur Ribeiro de Saboya. $\mathrm{Na}$ avaliação primária, apresentava-se consciente, porém com intenso quadro hemorrágico que foi contido por meio de ligadura da carótida externa bilateralmente sob anestesia geral. A avaliação secundária mostrou lesão ipsilateral do nervo facial nos seus ramos frontal e orbicular do olho gerando déficit motor nos músculos faciais correspondentes. Ao exame de tomografia computadorizada, pôde-se observar fratura cominutiva do côndilo mandibular direito. Optou-se pelo tratamento não-cirúrgico da fratura associado à terapia miofuncional orofacial. O projétil se encontrava na região de origem do músculo esternocleidomastoideo e foi preservado por não estar em intimidade com as estruturas articulares promovendo impedimento mecânico. Inicialmente, barras de Erich superior e inferior foram utilizadas para proporcionar tracionamento elástico maxilomandibular visando correção da oclusão que se apresentava alterada.

Os aspectos observados na avaliação fonoaudiológica, conforme protocolo de avaliação miofun- 
cional orofacial ${ }^{8}$, realizada após um mês e dez dias do acometimento foram:

- leve edema persistente e resposta dolorosa à palpação em região central do músculo esternocleidomastóideo direito, devido à presença do projétil próximo à região de inserção desse músculo;

- limitação do movimento de cabeça para o lado esquerdo tanto em inclinação quanto em rotação;

- déficit motor e sensitivo na região de terço médio e superior da hemi-face direita, com presença de paralisia facial acometendo músculos zigomáticos e orbicular do olho, lagoftalmo com consequente lacrimejamento e hiperemia do olho direito;

- dor à palpação nos músculos masseteres sendo grave à direita e moderada à esquerda, dor moderada nos músculos temporais bilateralmente e em esternocleidomastóideo do lado direito;

- mordida aberta anterior de $2 \mathrm{~mm}$, amplitude de abertura máxima em $30 \mathrm{~mm}$ com desvio em abertura de $7 \mathrm{~mm}$ para o lado direito (Figura 1), movimento de lateralidade direita $9 \mathrm{~mm}$ (Figura 2), lateralidade esquerda $2 \mathrm{~mm}$, protrusiva máxima em 1,5mm com abertura associada e desvio acentuado para direita de $9 \mathrm{~mm}$ (Figura 3).

- Aspectos funcionais: articulação da fala com desvio sistemático para direita associado à interposição lingual compensatória à esquerda, provocando distorção dos fones fricativos dentoalveolares inferiores [s] e [z]; mastigação limitada com poucos ciclos mastigatórios, preferencial à direita com complementação de amassamento do alimento com a língua contra o palato duro; deglutição com pressionamento de língua em região anterior compensatória à limitação de movimento livre da laringe devido à localização do projétil, observando-se lentidão do movimento e auxílio de elevação da laringe por movimento de hiperextensão anterior de cabeça.

\section{Procedimentos terapêuticos}

Foram realizadas oito sessões de terapia miofuncional orofacial, seguindo protocolo específico para fraturas de côndilo unilaterais ${ }^{8,9,15}$, acrescido de manobras específicas voltadas para o quadro de paralisia facial existente, com os seguintes objetivos: estimular e possibilitar a execução de movimentos mandibulares, impedindo a formação de tecido cicatricial restritivo na ATM envolvida; restabelecer mobilidade mandibular, evitando possíveis assimetrias ou limitações funcionais; estimular movimentos de abertura mandibular, organização

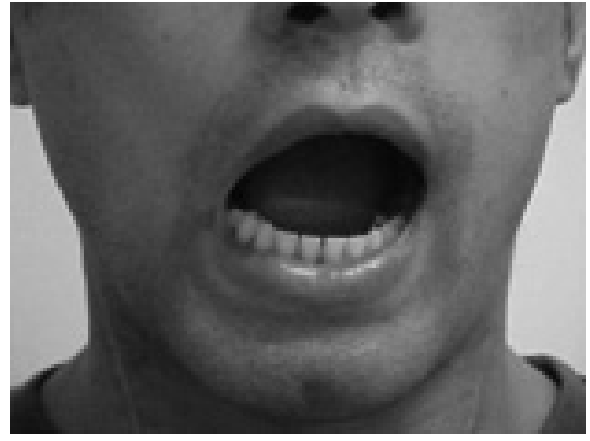

Figura 1 - Abertura máxima com desvio para o lado acometido

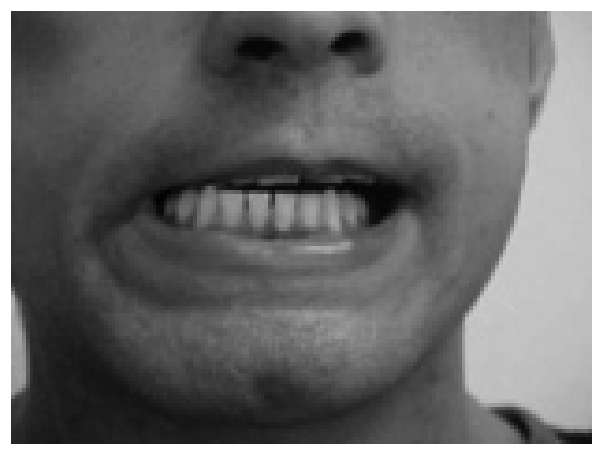

Figura 2 - Lateralidade para o lado acometido

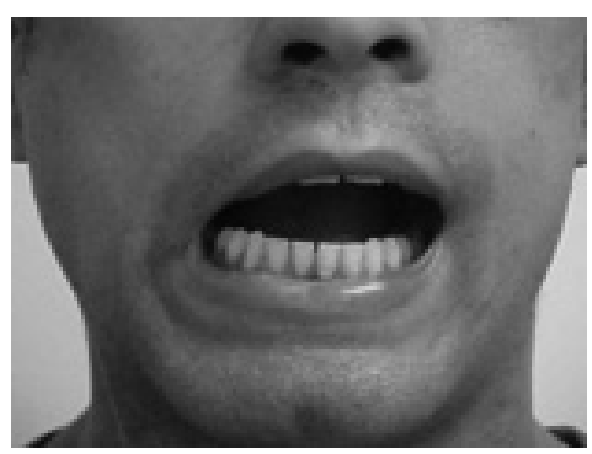

Figura 3 - Protrusiva com desvio e abertura associada

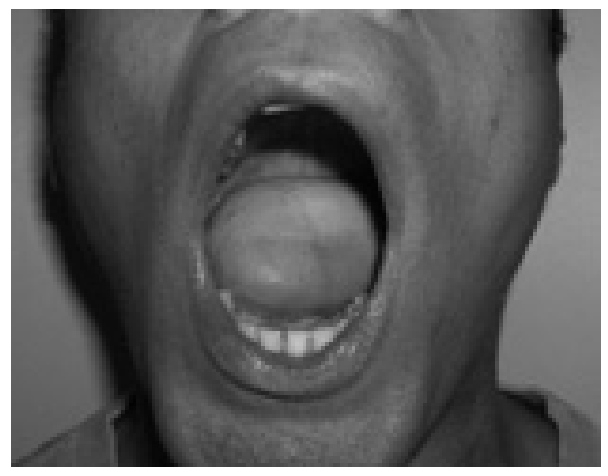

Figura 4: Abertura final 
de lateralidade e de protrusiva; reabilitação e estabilidade funcional quanto à fala, mastigação e deglutição.

Foram realizados: registro da avaliação inicial, frequência de terapia semanal, solicitação de assiduidade dos treinos em casa diariamente, três vezes por período; reavaliação após oito semanas ao término do tratamento sistemático.

O protocolo de atendimento realizado encontrase aqui detalhado:

1. Registro da amplitude dos movimentos mandibulares ao início e ao término de cada sessão buscando-se constatar a eficiência dos procedimentos realizados e controle da evolução a cada sessão.

2. Explicações e orientações quanto à retirada de apoios em mandíbula, não dormir em decúbito ventral, necessidade de alimentação amolecida inicialmente e direcionamento da mastigação. As orientações foram refeitas a cada sessão, e a dieta gradativamente retomada em termos de consistência.

3. Preparo da região escapular e cervical com massagens, alongamentos e manobras específicas visando liberação dos movimentos das estruturas envolvidas:

a. drenagem manual do edema em região do músculo esternocleidomastóideo visando aceleração da recuperação do processo inflamatório e aumento de oxigenação dos tecidos, com movimentos de compressão leves e descendentes;

b. alongamento e estimulação da musculatura do pescoço por meio de massagens em músculos cervicais posteriores e trapézios, seguido de movimentos dirigidos de lateralização e inclinação da cabeça;

c. manipulação passiva da laringe com movimentos lentos e leves lateralmente.

4. indução de mobilidade vertical da laringe por manipulação ativa com emissão de sons vocálicos passando de timbre grave para agudo sequencialmente;

5. Estimulação facial específica em musculatura acometida pela paralisia facial: orbicular do olho e músculos zigomáticos maior e menor do lado direito. Massagem indutora do movimento dos músculos zigomáticos, acompanhadas por solicitação de contração dos mesmos. Massagens indutoras em orbicular do olho direito em aproximação de fechamento e toques leves em região superior central e mesial desse músculo, acompanhadas por solicitação de fechamento forçado do olho, visando minimizar o lagoftalmo;
6. Massagens específicas de oxigenação e alongamento da musculatura levantadora da mandíbula: temporal e masseter do lado acometido, firmes e rotatórias contrárias à direção de contração das fibras. Alongamento de músculo masseter com massagem bidigital (interna e externa concomitante) em deslizamento diagonal e alongamento por vibração bidigital, tracionando o músculo em direção anterior e inferior;

7. Mobilidade mandibular leve (em torno de 10 a $15 \mathrm{~mm}$ ) visando oxigenação e controle muscular evitando-se desvios do percurso mandibular;

8. Movimentos mandibulares utilizando apoio da musculatura da língua. Movimentos de língua sequenciais de máxima elevação e abaixamento de ponta em protrusão máxima visando abertura espontânea da boca e movimento protrusivo associado; rotação e lateralização de língua no arco inferior com esforço para o lado esquerdo, visando movimento mandibular associado em lateralidade;

9. Abertura mandibular livre, movimentos mandibulares dirigidos e alavanca com apoio em dentes posteriores corrigindo desvios. Os movimentos mandibulares foram realizados por meio de:

a. abertura e fechamento livre; protrusivo com indução do contato incisal inferior em lábio superior e lateralidade para esquerda com o auxílio da rotação da língua;

b. aumento da abertura máxima, mesmo com desvio, mantendo a mandíbula no limite máximo obtido;

c. abertura forçada com colocação de espátulas ou dedo apoiado entre os dentes posteriores do lado acometido, mantendose sem forçar para que não ocorra contração muscular de proteção. A seguir, manobras de distração com o apoio das espátulas sempre nos dentes posteriores com movimento de alavanca lenta e gradativa, elevando-se a ponta livre dos palitos em direção superior;

d. movimento protrusivo, mantendo-se a contração na posição mais anterior conseguida sem desvio e lateralidade mandibular contralateral à lesão, mantendo-se nessas posições por alguns segundos;

e. ampliação e correção dos desvios no movimento de abertura da boca com abertura controlada por paradas no movimento, para estabilização da contração muscular.

10. Reorganização funcional: treinos sistemáticos da mastigação contralateral à fratura 
direcionando-se mecanismo de contração de bucinador e lateralização da língua unilateralmente induzindo movimento mandibular rotatório; aumento da amplitude e organização do movimento mandibular na fala por meio de pistas perceptivas e visuais; treinos dirigidos de deglutição com apoio de língua superior e movimento ondulatório, sem pressão em musculatura perioral e percepção do movimento de elevação suave da laringe;

11. Massagens e alongamentos da região cervical e cintura escapular.

\section{RESULTADOS}

A cada terapia é esperado que se obtenha em torno de 5 a $8 \mathrm{~mm}$ de aumento de abertura livre (com desvio) e 3 a $5 \mathrm{~mm}$ de abertura corrigida: comparando-se a medida de abertura ao início e ao final da terapia. O paciente, ao realizar o treino em casa durante a semana, conseguiu manter estabilidade desse ganho de amplitude, em torno de $3 \mathrm{~mm}$ por semana. Obtendo-se amplitude máxima próxima de $35 \mathrm{~mm}$, o treino passou a ser realizado três vezes ao dia garantindo-se assim a progressão dos resultados e estabilidade.
Após oito semanas de terapia miofuncional orofacial os resultados obtidos foram: redução dos mecanismos compensatórios; remissão da sintomatologia dolorosa; recuperação da mobilidade muscular do terço médio do lado acometido; ganho de $18 \mathrm{~mm}$ na abertura mandibular máxima, passando de $30 \mathrm{~mm}$ para $48 \mathrm{~mm}$, sem desvio (Tabela 1, figura 4); estabilidade na amplitude máxima dos movimentos mandibulares conseguida e maior liberdade nos movimentos mandibulares obtendo-se lateralidade direita em $8 \mathrm{~mm}$, esquerda $7 \mathrm{~mm}$ (Tabela 1) e protrusiva em $4 \mathrm{~mm}$ sem desvio; reorganização da mastigação com melhora do giro mandibular, aumento dos ciclos mastigatórios e eliminação da utilização de amassamento do alimento com a língua; adequação da deglutição com remoção das pressões atípicas e suavização do movimento de elevação da laringe; melhora na articulação da fala e eliminação das distorções, embora se mantenha discreto desvio para direita, em torno de $2 \mathrm{~mm}$.

Não foi obtida melhora significante quanto ao músculo orbicular do olho do lado direito, mantendose a dificuldade em fechá-lo com consequente lacrimejamento e hiperemia. Por indicação da equipe médica foi realizada implantação de peso de ouro em pálpebra superior direita para correção do lagoftalmo ${ }^{16} \mathrm{com}$ bom resultado funcional.

Tabela 1 - Progressão da amplitude de abertura e lateralidade mandibular esquerda (em $\mathrm{mm}$ ) durante o tratamento

\begin{tabular}{lcc}
\hline & Abertura máxima $(\mathbf{m m})$ & Lateralidade esquerda $(\mathbf{m m})$ \\
\hline Avaliação & 30 & 2 \\
$2^{\text {a }}$ semana & 38 & 4 \\
$3^{\text {a }}$ semana & 41 & 6 \\
$4^{\text {a }}$ semana & 44 & 6 \\
$8^{\text {a }}$ semana & 48 & 7 \\
\hline
\end{tabular}

\section{DISCUSSÃo}

Os ferimentos por projéteis de arma de fogo na face geralmente promovem grandes deformidades e alterações funcionais importantes, principalmente quando atingem a ATM ou danificam estruturas nervosas como o nervo facial ${ }^{5,17}$. O tratamento das fraturas condilares ainda é bastante controverso na literatura ${ }^{12,13,18-21}$, porém resultados funcionais aceitáveis clinicamente são encontrados tanto no tratamento cirúrgico quanto no tratamento não-cirúrgico $0^{911,20-22}$.

Existem na literatura contra-indicações absolutas para a redução aberta e fixação rígida das fraturas condilares, sendo a fratura intracapsular e cominutiva a principal delas ${ }^{23}$, uma vez que podem desenvolver extensa reabsorção local, em conseqüência do descolamento periostal necessário no período trans-operatório ${ }^{14}$.

Quanto aos resultados funcionais, estudo com vítimas de ferimento por projétil de arma de fogo na mandíbula aponta em média $28 \mathrm{~mm}$ de abertura bucal máxima aos dois meses pós-trauma e $36 \mathrm{~mm}$ no quinto mês pós trauma ${ }^{24}$. Estudo referente aos resultados de pacientes com fraturas condilares intracapsulares tratados de modo não-cirúrgico encontraram, em $74 \%$ dos casos com fraturas cominutivas, importante alteração na excursão condilar 
e limitação dos movimentos mandibulares, quando comparado com o lado não afetado ${ }^{25}$. Os mesmos autores, em $2005^{26}$ observaram que $30 \%$ dos casos de fratura condilar tratados de modo não-cirúrgico, apresentavam desvio lateral durante a abertura da boca, crepitação e alteração oclusal.

Por outro lado, encontra-se na literatura que a restauração funcional ocorre de maneira satisfatória em pacientes jovens com fratura condilar quando estes são tratados com ortopedia funcional ou exercícios ${ }^{27,28}$. A abordagem fonoaudiológica miofuncional orofacial é apontada como eficiente tratamento complementar tanto em fraturas de côndilo, de processo coronóide, processo pterigóide, e fraturas múltiplas de mandíbula, devolvendo a amplitude e organizando os movimentos mandibulares, além de restabeler as funções orofaciais, evitando-se assim o risco de hipomobilidade e redução funcional $\left.\right|^{4,9,11,15,27,29}$. Os resultados do presente trabalho mostram recuperação da amplitude máxima de abertura e correção dos desvios em lateralidade apresentando, portanto melhor resultado e em menor período de tempo quando comparado às citações anteriores ${ }^{24-26}$.

Quanto à proposta fonoaudiológica miofuncional orofacial descrita para esse caso, foi desenvolvida uma programação específica envolvendo manobras e manipulações na região orofacial e cervical, buscando-se restabelecer a funcionalidade do sistema estomatognático e evitar sequelas $4,9,11,29$, respeitando-se as limitações do paciente em função do quadro associado à presença do projétil. A região cervical foi trabalhada durante o processo terapêutico devido aos mecanismos adaptativos compensatórios utilizados pelo paciente em decorrência do edema e desconforto provocados pela presença do projétil, principalmente quanto a modificação do movimento da laringe durante a deglutição. Movimentos voltados para a musculatura cervical e laríngea como lateralização manual, elevação por meio de vocalizações, hiperextensão e hiperflexão da cabeça, foram estimulados juntamente com massagens e alongamento nos músculos cervicais, esternocleidomastóideos e trapézio buscando promover a oxigenação e organização funcional|8,9,15,29, mesmo que parcial da situação muscular envolvida nos mecanismos compensatórios necessários nesse tipo de caso.

Os movimentos mandibulares foram incentivados buscando-se a simetria do movimento e aumento de sua amplitude. Para tal foram utilizadas manipulações passivas com o auxílio dos movimentos de língua, considerando-se que essa é uma importante estrutura de apoio e direcionamento na realização dos movimentos mandibulares devido à inserção de sua musculatura extrínseca. Assim, por meio de exercícios de protrusão e elevação da língua fora da boca estimula-se a abertura mandibular acompanhada por movimento mandibular protrusivo. Com o abaixamento da língua fora da boca se intensifica a abertura. A lateralização da língua, fora ou dentro da boca, estimula os movimentos de lateralidade mandibular e a rotação, os movimentos sequenciais ${ }^{8,15,29}$.

Além dos movimentos mandibulares ativos, coordenados e direcionados por estímulos visuais e tácteis sinestésicos apresentados, foram também utilizadas algumas manobras com espátulas principalmente visando aumento da amplitude e correção dos desvios. A utilização da manobra com espátulas intraoral, em caso de fratura da cabeça da mandíbula, só deve ser realizada com apoio dessas em região dos últimos molares presentes do paciente. As espátulas nunca devem ser colocadas na região anterior, uma vez que a utilização nessa posição leva à redução de altura funcional posterior devido à direção da alavanca, podendo provocar maior deslocamento do fragmento nos casos de fraturas. $\mathrm{O}$ apoio em dentes posteriores e a pressão da alavanca realizada no sentido superior buscam alongamento da musculatura levantadora da mandíbula e ganho de altura posterior com liberação de espaço em região da cabeça da mandíbula. Os cuidados descritos nessas manobras são fundamentais para se evitar qualquer sobrecarga na articulação não acometida e também para que a musculatura não responda com contração de proteção, mascarando a efetividade do trabalho ${ }^{29}$.

A mastigação, deglutição e articulação da fala foram também trabalhadas criteriosamente uma vez que tais funções encontravam-se alteradas e sua organização atua como estímulo contínuo. Foi proposto treino mastigatório com alimentos macios inicialmente passando-se para texturas variadas, buscando-se organização e retomada gradativa da alimentação. A solicitação quanto ao padrão unilateral contralateral à fratura buscou fornecer estímulo de tração e lateralidade combinadas à estimulação do mecanismo de contração do músculo bucinador, orbicular da boca e lateralização de língua. Assim foram fornecidos estímulos facilitadores de obtenção do movimento mandibular associados à organização do bolo alimentar, ${ }^{8,9,15,29}$. Especificamente nesse caso, a deglutição necessitou de enfoque terapêutico em função da limitação do movimento de laringe.

As paralisias faciais traumáticas são a segunda causa mais frequente de suas etiologias, ficando atrás somente dos casos ditos idiopáticos. Dentre as causas desses traumas mais comuns estão os provocados por traumatismos cranianos seguidos por projéteis de arma de fogo ${ }^{1}$. A proposta 
terapêutica apresentada quanto a esse item mostrou-se eficiente em relação ao terço médio da face, porém não foi suficiente quanto ao lagoftalmo, tornando-se necessário procedimento de implantação de peso de ouro conforme descrito em trabalho anterior ${ }^{16}$.

Essa proposta interdisciplinar, com ênfase em terapia miofuncional orofacial, desenvolvida para pacientes acometidos por fratura condilar cominutiva, visa não só aumentar a abertura da boca e liberar os movimentos da mandíbula, mas principalmente propiciar melhores condições de funcionali- dade e harmonia do complexo e dinâmico sistema estomatognático.

\section{CONCLUSÃO}

O tratamento conservador da fratura condilar cominutiva por projétil de arma de fogo, voltado para terapêutica miofuncional orofacial resultou na reabilitação funcional da mandíbula e da face dirigindo e organizando os movimentos, assim como estimulando a adequação das funções estomatognáticas, buscando evitar a progressão de possíveis sequelas.

\begin{abstract}
Background: firearm wounds are relatively frequent and show high incidence at the head and face area. Temporomandibular joint may be involved, and also some important anatomic structures as the facial nerve directing the need for an interdisciplinary team in order to promote an efficient treatment. Procedures: a case report related to a comminuted condyle fracture caused by firearms with a nonsurgical treatment associated to myofunctional therapy. The patient was referred to Speech and Language Pathologist after the conduct of oral and maxillofacial surgery team, without removing the bullet, lodged superficially near the origin of the sternocleidomastoid muscle on the right side; with comminuted condyle fracture and facial nerve damage. Myofunctional evaluation found an important reduction in the mandibular movement amplitude with severe deviations as for the affected side, no contralateral laterality, muscle pain; paralysis and paresthesia on the right side of the face. Myofunctional therapy followed a specific protocol for facial trauma including: drainage of edemas, specific ipsilateral manipulations on jaw muscles; correction and enlargement of the mandibular movements, specific procedures related to facial paralysis and directed functional reorganization. Results: after eight therapy sessions we obtained: mandibular movements with adequate amplitude and symmetry, chewing reorganization, adequacy of swallowing and speech, remission of painful symptoms and remission of paralysis in the medium third of the face. Conclusion: the conservative treatment for comminuted condyle fracture through myofunctional therapy resulted in functional rehabilitation of the jaw and face, directing the movements and stimulating the adequacy of the sthomatognatic functions.
\end{abstract}

KEYWORDS: Temporomandibular Joint; Fractures, Comminuted; Wounds, Gunshot; Rehabilitation; Myofunctional Therapy

\section{REFERÊNCIAS}

1. Pereira CCS, Jacob RJ, Takahashi A, Shinohara EH. Fratura mandibular por projétil de arma de fogo. Rev. cir. traumatol. buco-maxilo-fac. 2006; 6(3):39-46.

2. Maliska MC; Lima Júnior SM; Gil JN. Analysis of 185 maxillofacial fractures in the state of Santa Catarina, Brazil. Braz Oral Res.2009 Jul-Sep;;23(3):268-74.

3. Wulkan M, Parreira Júnior JG, Botter DA. Epidemiologia do trauma facial. Rev Assoc Med Bras. 2005;51(5):290-5.
4. Oliveira DM de, Vasconcellos RJH, Laureano Filho JR, Cypriano RV. Fracture of the coronoid and pterygoid processes by firearms: case report. Braz. Dent. J 2007;18(2):168-70.

5. He D, Zhang Y, Ellis E. Panfacial fractures: analysis of 33 cases treated late. J Oral Maxillofac Surg. 2007; 65(12):2459-65.

6. Pinna BR; Testa JRG; Fukuda Y. Estudo de paralisias faciais traumáticas: análise de casos clínicos e cirúrgicos. Rev. Bras. Otorrinolaringol. 2004; 70(4):479-82.

7. Zachariades N, Mezitis M, Mourouzis C, Papadakis D, Spanou A. Fractures of the mandibular condyle: 
a review of 466 cases. Literature review, reflections on treatment and proposals. J Craniomaxillofac Surg. 2006;34(7):421-32.

Early mobilization is the key in treating condylar fractures.

8. Bianchini EMG. Traumas de Face: Atuação fonoaudiológica, caracterização, proposta terapêutica e resultados. In: Comitê de Motricidade Oral SBFa. (Org.). Motricidade Orofacial: Como atuam os especialistas. São José dos Campos: Pulso Editorial; 2004. p. 107-14.

9. Bianchini, EMG; Mangilli, L. D.; Marzotto, SR; Nazário, D. Pacientes acometidos por trauma da face: caracterização, aplicabilidade e resultados do tratamento fonoaudiológico específico. Revista CEFAC, 2004;6(4):388-95.

10. Bianchini EMG. Características funcionais dos pacientes com disfunções e alterações da ATM. In: Bianchini EMG. organizadora. Articulação Temporomandibular: Implicações, Limitações e Possibilidades Fonoaudiológicas, 2a Ed. Carapicuíba: Pró-Fono; 2010. p. 255-77.

11. Sakae EK, Barroso, MVVS, Hiraki PY, Moura T, D'Agostinho L. Tratamento conservador de fratura cominuta de mandíbula ocasionada por arma de fogo: relato de caso. Rev Soc Bras Cir Craniomaxilofac. 2008;11(3,supl):16-16.

12. Filho LI, Pavan AJ, Camarini ET, Tondin GM. Tratamento das fraturas de côndilo mandibular: cirúrgico ou conservador? Rev Cir Traumatol BucoMaxilo-Fac. 2005; 4:17-22.

13. Landes CA, Lipphardt R. Prospective evaluation of a pragmatic treatment rationale: open reduction and internal fixation of displaced and dislocated condyle and condylar head fractures and closed reduction of non-displaced, non-dislocated fractures Part II: high condilar and condilar head fractures. Int J Oral Maxillofac Surg 2006; 35 (2): 115-26.

14. lizuka T, Lindqvist $C$, Hallikainen D, Mikkonen $P$, PaukkuP. Severe bone resorption and osteoarthrosis after miniplate fixation of high condylar fractures. A clinical and radiologic study of thirteen patients. Oral Surg Oral Med Oral Pathol. 1991; 72(4): 400-7.

15. Bianchini EMG. Ajuda fonoaudiológica. In: Bianchini EMG. organizadora. Articulação temporomandibular: implicações, limitações e possibilidades fonoaudiológicas. 2a Ed. Carapicuiba: Pró-Fono, 2010. p.321-61.

\section{RECEBIDO EM: 15/01/2010 \\ ACEITO EM: 02/08/2010}

Endereço para correspondência:

Esther M.G.Bianchini

Alameda Jaú, 1767, cj 51, São Paulo - SP

CEP: 11420-002

E-mail: esther.bianchini@uol.com.br
16. Lessa S, Nanci M, Flores E, Sebastiá R. Tratamento do lagoftalmo paralítico com a utilização do implante de peso de ouro recoberto pela aponeurose do músculo levantador palpebral. Rev Bras Oftalmol. 2009;68(1):30-6.

17. Kelly $P$, Drago CJ. Surgical and prosthodontic treatment of a patient with significant trauma to the middle and lower face secondary to a gunshot wound: a clinical report. J Prosthodont. 2009 Oct;18(7):626-37.

18. Muller RV, Czerwinski M, Lee C, Kellman RM. Condylar fracture repair: use of the endoscope to advance traditional treatment philosophy. Facial Plast Surg Clin North Am. 2006;14(1):1-9.

19. Mortellaro C; Rimondini L; Farronato G; Garagiola $\mathrm{U}$; Varcellino V; Berrone M. Temporomandibular disorders due to improper surgical treatment of mandibular fracture: clinical report.J Craniofac Surg. 2006 Mar; 17(2):373-82.

20. Gorzelnik L; Kozlovsky E Bicortical extraoral plating of mandibular fractures.Atlas Oral Maxillofac Surg Clin North Am. 2009 Mar; 17(1):35-43.

21. He D, Yang C, Chen M, Jiang B, Wang B. Intracapsular condylar fracture of the mandible: our classification and open treatment experience. J Oral Maxillo Surg 2009 Aug;67(8):1672-9.

22. Stiesch-Scholz M, Schmidt S, Eckardt A. Condylar motion after open and closed treatment of mandibular condilar fractures. J Oral Maxillofac Surg. 2005;63(9):1304-9.

23. Zide MF, Kent JN. Indications for open reduction of mandibular condyle fractures. J Oral Maxillofac Surg. 1983; 41:89-98.

24. Neupert EA III, Body SB. Retrospective analysis of low velocity gunshot wounds to the mandible. Oral Surg Oral Med Oral Pathol. 1991;72(4):383-7.

25. Hlawitschka M, Eckelt U. Assessment of patients treated for intracapsular fractures of the mandibular condyle by closed techniques. J Oral Maxillofac Surg. 2002;60(7):784-91.

26. Hlawitschka M, Loukota R, Eckelt U. Functional and radiological results of open and closed treatment of intracapsular (diacapitular) condilar fractures of the mandible. Int J Oral Maxillofac Surg. 2005;34(6):597-604.

27. Prayer Galletti S, Marchesi A, Cudia G. The functional therapy of condilar fractures: a Kinesiographic follow-up. Minerva Stomatol 1990; 39(10): 849-53.

28. Kahl B, Gerlach KL. Functional treatment after condilar fractures with and without an activator. Fortschr Kieferorthop 1990; 51(6): 352-60.

29. Marzotto SR, Bianchini EMG. Anquilose temporomandibular bilateral: aspectos fonoaudiológicos e procedimentos clínicos. Revista CEFAC 2007; 9(3): 358-66. 